\title{
Heterodyne dual-polarization epi- detected CARS microscopy for chemical and topographic imaging of interfaces
}

Wolfgang Langbein, Dafydd Sion Harlow, David Regan, lestyn Pope, Paola Borri

Wolfgang Langbein, Dafydd Sion Harlow, David Regan, lestyn Pope, Paola Borri, "Heterodyne dual-polarization epi-detected CARS microscopy for chemical and topographic imaging of interfaces," Proc. SPIE 10890, Labelfree Biomedical Imaging and Sensing (LBIS) 2019, 1089003 (4 March 2019); doi: $10.1117 / 12.2507636$ 


\title{
Heterodyne dual-polarization epi-detected CARS microscopy for chemical and topographic imaging of interfaces
}

\author{
Wolfgang Langbein ${ }^{\mathrm{a}}$, Dafydd Sion Harlow ${ }^{\mathrm{b}}$, David Regan ${ }^{\mathrm{a}}$, Iestyn Pope ${ }^{\mathrm{b}}$, and Paola Borri ${ }^{\mathrm{b}}$

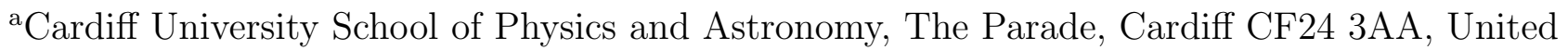 \\ Kingdom \\ ${ }^{\mathrm{b}}$ Cardiff University School of Biosciences, Museum Avenue, Cardiff CF10 3AX, United \\ Kingdom
}

\begin{abstract}
We present a label-free vibrational microscopy technique recently developed by us, which offers backgroundfree chemically-specific image contrast, shot-noise limited detection, and phase sensitivity enabling topographic imaging of interfaces. The technique features interferometric heterodyne detection of coherent anti-Stokes Raman scattering (CARS) in epi-geometry, as well as multi-modal acquisition of stimulated Raman scattering and forward-emitted CARS intensity in the same instrument. As an important biologically-relevant application, epi-detected heterodyne CARS imaging of individual lipid bilayers is demonstrated. We show that we can resolve a single lipid bilayer, distinct from a double bilayer, and measure the phase of its susceptibility, which provides information about the topography of the bilayer with nanometer resolution. As an additional application example, we show imaging of silicon oil droplets surrounded by an aqueous environment at the glass-water interface, where three different signal generation pathways are distinguished. Our epi-detected heterodyne CARS microscope setup thus paves the way to exciting new experiments pushing the sensitivity and resolution limits of vibrational microscopy to the nanoscale.
\end{abstract}

Keywords: coherent Raman scattering, multiphoton microscopy, heterodyne interferometry, lipid bilayers

\section{INTRODUCTION}

Coherent Raman Scattering (CRS) micro-spectroscopy has gained increasing attention over the last two decades, and is becoming an established multiphoton microscopy technique offering label-free chemically-specific imaging at video rates combined with sub-micron three-dimensional spatial resolution. ${ }^{1-3}$ To understand the working principle of CRS, let us recall that Raman scattering is an inelastic interaction of light with vibrating chemical bonds which gives rise to scattered light at a different optical frequency (color) compared to the incident field. The frequency shift is equal to the frequency of the vibration, which in turn depends on the type of chemical bond. This scattering phenomenon usually produces a very weak signal, but can be strongly enhanced in CRS when two short laser pulses (denoted as pump and Stokes of optical frequencies $\omega_{\mathrm{P}}$ and $\omega_{\mathrm{S}}$ respectively) are used to coherently drive the vibrations at frequency $\omega_{\mathrm{P}}-\omega_{\mathrm{S}}$, such that Raman scattered light from coherently driven molecular vibrations in the focal volume constructively interferes. As a result, chemically specific label-free CRS microscopy at imaging speeds compatible with studying living specimen has become possible and was applied to many biological samples, with particular emphasis on imaging lipids owing to the large number of identical $\mathrm{CH}$ bonds in their acyl chain..$^{2,4-7}$

A common implementation of CRS detects the frequency up-shifted Raman scattering of the higher excitation frequency (pump), called coherent anti-Stokes Raman scattering (CARS). This signal detection is free from excitation background since the CARS optical frequency $\omega_{\mathrm{CARS}}$ is spectrally well separated from the frequency of the exciting pump and Stokes pulses, following the relation $\omega_{\mathrm{CARS}}=2 \omega_{\mathrm{P}}-\omega_{\mathrm{S}}$. A simple and widely adopted way to measure CARS is then to detect its intensity, proportional to the modulo square of the field, and thus proportional to the modulo square of the non-linear CRS susceptibility. However, in this way, the vibrationally

Further author information: Send correspondence to borrip@cf.ac.uk

Label-free Biomedical Imaging and Sensing (LBIS) 2019, edited by Natan T. Shaked,

Oliver Hayden, Proc. of SPIE Vol. 10890, 1089003 - @ 2019 SPIE

CCC code: $1605-7422 / 19 / \$ 18 \cdot$ doi: $10.1117 / 12.2507636$

Proc. of SPIE Vol. 10890 1089003-1 
resonant and non-resonant parts of the complex non-linear CRS susceptibility interfere, giving rise to an asymmetric spectral lineshape for a resonant response. ${ }^{8}$ The non-resonant part also causes a background, reducing image contrast for microscopy applications. Note that the CRS susceptibility is actually the quantity of interest, being proportional to the local material composition.

Many technical realizations of CRS microscopy have been proposed to remove, suppress, or account for the non-resonant background in the nonlinear susceptibility. For example, when acquiring the CARS intensity over a wide spectral range, it is possible to retrieve the phase of the susceptibility.$^{9-14}$ However, this requirement limits the imaging speed, prompting the development of alternative methods. Of particular interest is the idea to use an interferometric detection to measure the CARS field directly in amplitude and phase, thus retaining its full information content.${ }^{15-17}$ In this way, one can directly isolate the imaginary part of the complex susceptibility, which is given by the resonant response only, and is similar to the signal measured in spontaneous Raman scattering. However, achieving interferometric stability between the signal and the reference beams is challenging.

Recently, we have developed a multimodal microscope featuring a novel CARS interferometric, dualpolarization heterodyne detection scheme in epi-geometry with balanced diode detection, providing backgroundfree chemically specific image contrast, shot-noise limited detection, and phase sensitivity. ${ }^{18}$ Notably, in this implementation the epi-detected signal phase provides direct topographic information on the axial position of the sample with nanometer precision. A back-to-back comparison between stimulated Raman scattering (SRS), forward-detected CARS intensity (F-CARS), and epi-detected heterodyne CARS (eH-CARS) was shown on a set of polystyrene beads in agarose gel. Through this comparison, we demonstrated that eH-CARS imaging is free from the backgrounds limiting intensity-based forward CARS as well as SRS. As an important biologically relevant application, we also demonstrated eH-CARS imaging of individual lipid bilayers with high contrast and topographic sensitivity.

In this paper, we further showcase the application of our eH-CARS technique to image planar lipid bilayers on a glass substrate, notoriously pushing CRS microscopy to its detection limits. We show that we can resolve a single lipid bilayer, distinct from a double bilayer, and measure the phase of its susceptibility, which provides information about the topography of the bilayer with nanometer resolution. As an additional application example, we show imaging of silicon oil droplets surrounded by an aqueous environment at the glass-water interface, where phase variations in this case are related to the change in the material susceptibility from oil to agarose, and the surface topography of the droplets.

\section{EXPERIMENTAL SET-UP}

\subsection{Heterodyne epi-detected CARS}

The setup is described in detail in Ref. 18. Briefly, Pump, Stokes and reference beams of about 150 fs duration at $\nu_{\mathrm{L}}=80 \mathrm{MHz}$ repetition rate are provided by a Ti:Sa pulsed laser source which is frequency-doubled to pump an optical parametric oscillator (OPO), providing a tuneable signal beam in the wavelength range $490-750 \mathrm{~nm}$, and a corresponding idler in the range $930-2500 \mathrm{~nm}$. To drive vibrational resonances in the $\mathrm{CH}$-stretch region around $3000 \mathrm{~cm}^{-1}$, we use center wavelength of $820 \mathrm{~nm}$ for the a Pump, $1080 \mathrm{~nm}$ for the Stokes, and $660 \mathrm{~nm}$ for the signal used as heterodyne reference, which is phase- and wavelength-locked to the generated CARS field through the phase-preserving chain in the OPO.${ }^{17}$ We used spectral focussing, applying an equal linear chirp to pump and Stokes pulses, hence creating a constant instantaneous frequency difference (IFD) between them which selectively drives a vibrational excitation of interest. The spectral resolution is given by the Fourier-limit of the temporal envelope of the chirped pulses, which can be can be elongated to a few picoseconds ${ }^{19,20}$ corresponding to spectral resolutions in the $20 \mathrm{~cm}^{-1}$ range. As previously demonstrated, a simple and efficient control of the linear chirp of Pump and Stokes pulses is obtained when they travel through glass blocks of known group-velocity dispersion ${ }^{19,21}$ We used here H-ZF52A and SF57 glass blocks of selected lengths, and a motorized delay line in the Pump beam path to tune the IFD. The reference pulse is also chirped by glass blocks to match the chirp of the CARS signal at its interference at the detector.

For heterodyne detection, the Stokes beam was frequency up-shifted by the radiofrequency $\nu_{\mathrm{s}}=77 \mathrm{MHz}$ using an acousto-optic modulator (AOM) driven at $\nu_{\mathrm{s}}$. Pump and Stokes pulses are recombined and enter a commercial inverted microscope stand, and are focussed onto the sample by a high numerical aperture (NA) microscope 


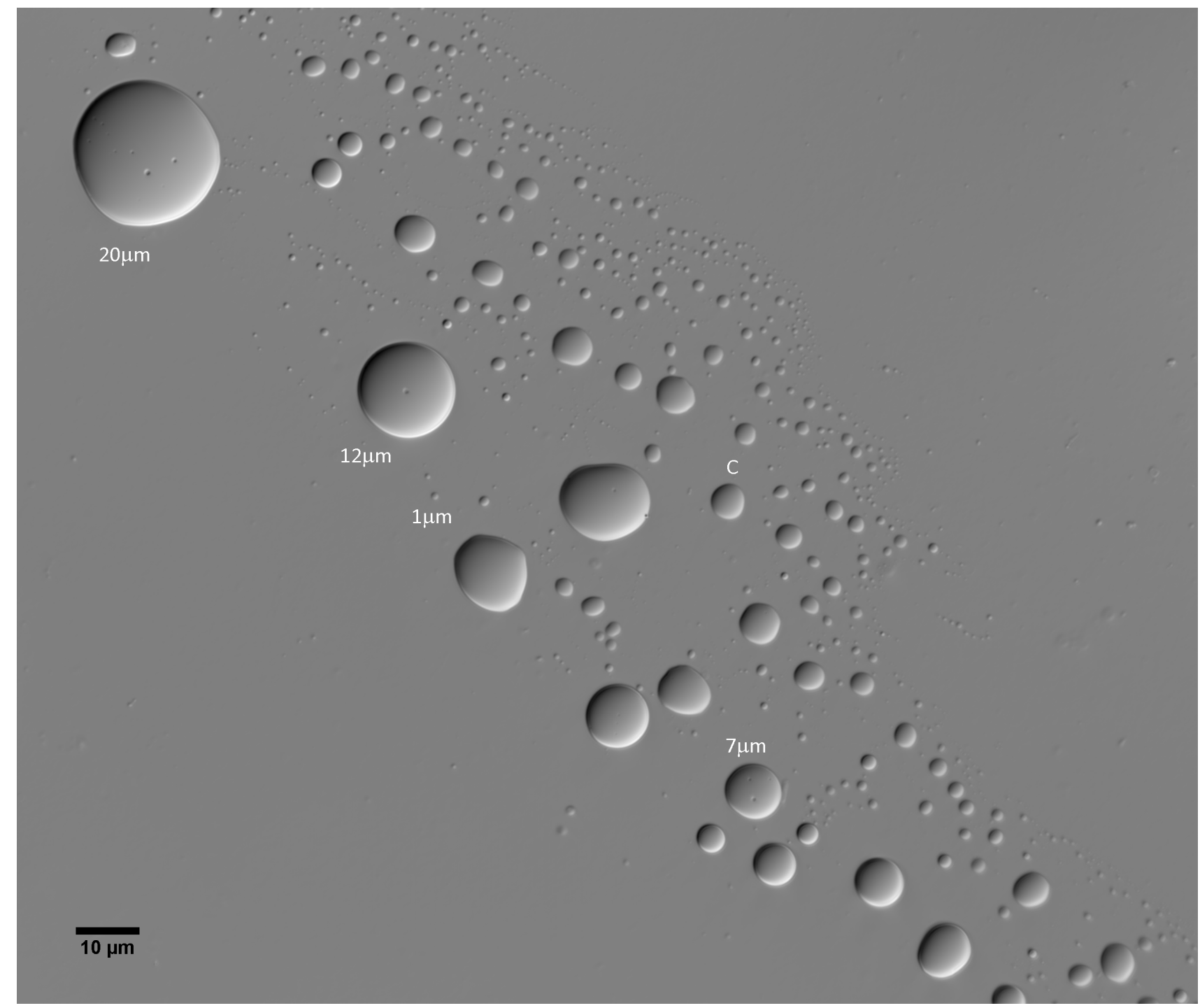

Figure 1. DIC differential interference contrast image $I_{\mathrm{C}}$ of the investigated sample of silicone oil droplets on a glass-water ( $0.25 \%$ agar) interface. Gray scale from -0.1733 (black) to 0.1744 (white). Lateral scale as given by scale bar. Specific droplets or regions are labelled for reference.

objective, either a $60 \times$ magnification water-immersion objective of 1.27NA (Nikon CFI plan apochromat lambdaS, MRD07650), for the measurements on lipid droplets, or a $100 \times$ magnification oil-immersion objective of $1.45 \mathrm{NA}$ (Nikon CFI plan apochromat lambda, MRD01905), for the measurements on planar lipid bilayers. The sample is positioned with respect to the objective by scanning an $x y z$ sample stage with nanometric position accuracy. eH-CARS is collected in epi-geometry by the same objective used in excitation, and overlapped with the reference beam. The resulting interference between the CARS and reference fields is detected by balanced photodiodes, rejecting the classical noise of the reference, and reaching shot noise limitation. The interference is detected at the beat note $\nu_{\mathrm{L}}-\nu_{\mathrm{s}}=3 \mathrm{MHz}$ using a dual-input dual-channel lock-in amplifier (ZI HF2), providing amplitude and phase components.

\subsection{Differential interference contrast}

Differential interference contrast (DIC) images were taken using the $60 \times$ water-immersion objective, and a 1.34NA oil condenser. Illumination was provided by a halogen tungsten lamp (V2-A LL 100W; Nikon), followed by a Schott BG40 filter to remove infra-red wavelengths outside the operating range of the DIC polarizers. A 


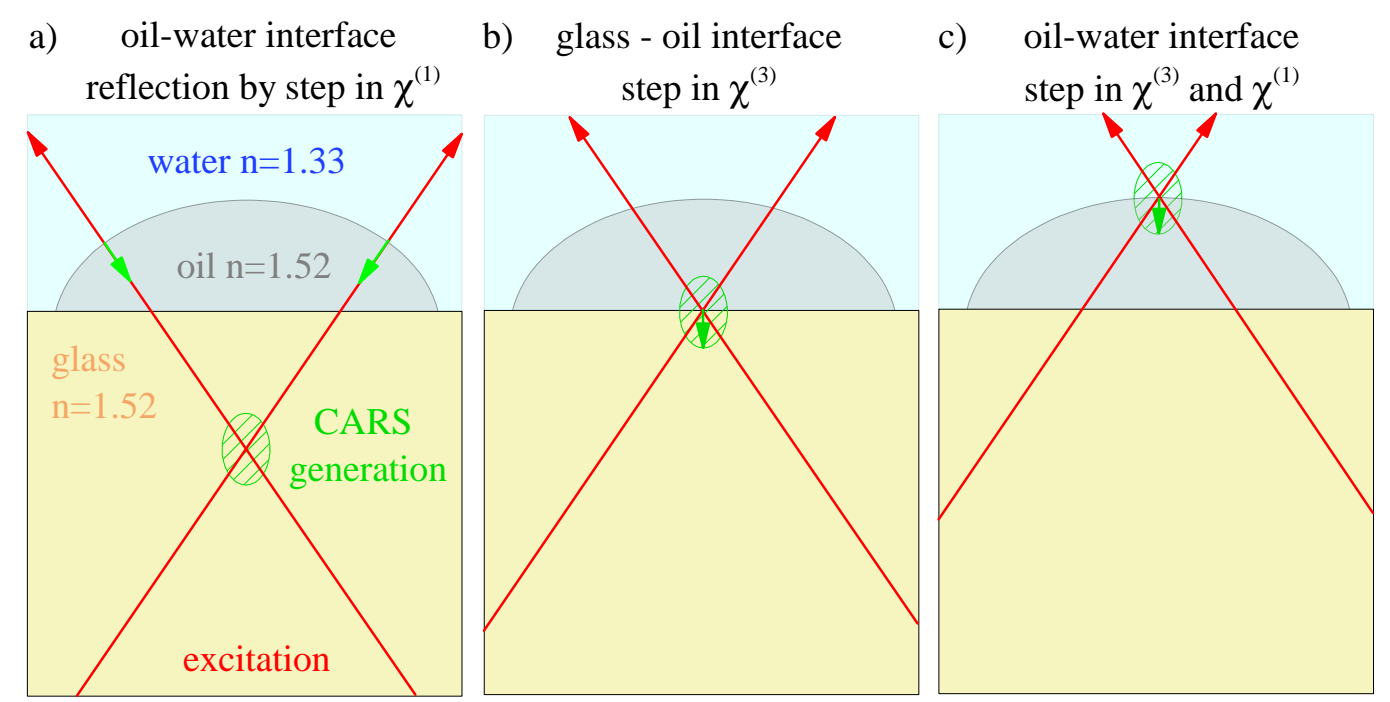

Figure 2. Sketch of eH-CARS signal generation pathways in the oil droplet samples. a) generation in glass, confocal reflection by droplet. b) generation by the step in $\chi^{(3)}$ between glass and oil. c) generation by the step in $\chi^{(3)}$ between oil and water, and reflection of the signal generated in oil by the oil-water interface.

de-Sénarmont compensator (T-P2 DIC Polarizer HT; Nikon) was used for offset phase adjustment, followed by a Wollaston prism (T-C DIC Module High NA N2 Oil; Nikon) in the condenser unit, a Nomarski prism after the objective (N2 60x-IV; Nikon), and a linear polarizer (Ti-A-E DIC Analyzer Block; Nikon) in the filter turret. DIC images were acquired using a scientific CMOS (PCO.edge 5.5; PCO, Germany). An average over 128 frames was acquired with the angle of the de-Senarmont polarizer set at $\pm 15^{\circ}$, yielding $I_{ \pm}$. The two images were then combined to a contrast image $I_{\mathrm{C}}=\left(I_{+}-I_{-}\right) /\left(I_{+}+I_{-}\right)$, in which uneven illumination and detection, as well as transmission, are compensated.

\section{RESULTS AND DISCUSSION}

\subsection{Oil droplets on glass}

Here we investigate silicone oil droplets on a glass surface, in water $(0.25 \%$ agar $)$. The sample was prepared as follows. The coverslip glass and microscope slide was cleaned by scrubbbing with acetone and cleanroom paper, then sonicating three times in acetone, toluene and hydrogen peroxide. A gasket was placed in the centre of the microscope slide and the void was filled with $40 \mu \mathrm{L}$ of $0.25 \%$ agarose in DI water. Agarose was used to stabilize the position of the oil droplets. $5 \mu \mathrm{L}$ of degassed Silicone oil was then placed on the edge of the circular gasket void, on top of the agarose. The gasket was then covered with the coverslip and pressed gently to make a seal. Clear nail varnish was used to seal the edges of the coverslip. The sample consisted of some oil droplets in contact with the coverslip glass covered by agarose.

The DIC contrast of the droplet region investigated, focussed at the glass surface, is shown in Fig. 1. DIC provides a shadow cast impression, where one can appreciate the three-dimensional structure of the sample. We see a range of different droplet sizes, attached to the glass surface. Larger droplets show a non-spherical shape, sometimes also exhibiting small bubbles. The droplets investigated in the following are indicated by their approximate diameter.

We took three-dimensional eH-CARS measurements on a few of these droplets, ranging in size from about $1 \mu \mathrm{m}$ to about $20 \mu \mathrm{m}$. These are indicated in Fig. 1 by the labels. Measurements were taken at an IFD of $2904 \mathrm{~cm}^{-1}$, which is at the largest resonance peak of the silicone oil. Let us first discuss the eH-CARS signal generation pathways as sketched in Fig. 2. Epi-detection requires either a reflection of the CARS signal generated in forward direction, or CARS signal directly generated in backward direction. The latter requires a step in the CARS third-order susceptibility $\chi^{(3)}$, while the former is created by a step in the refractive index, that is, a step in the first-order susceptibility $\chi^{(1)}$. 

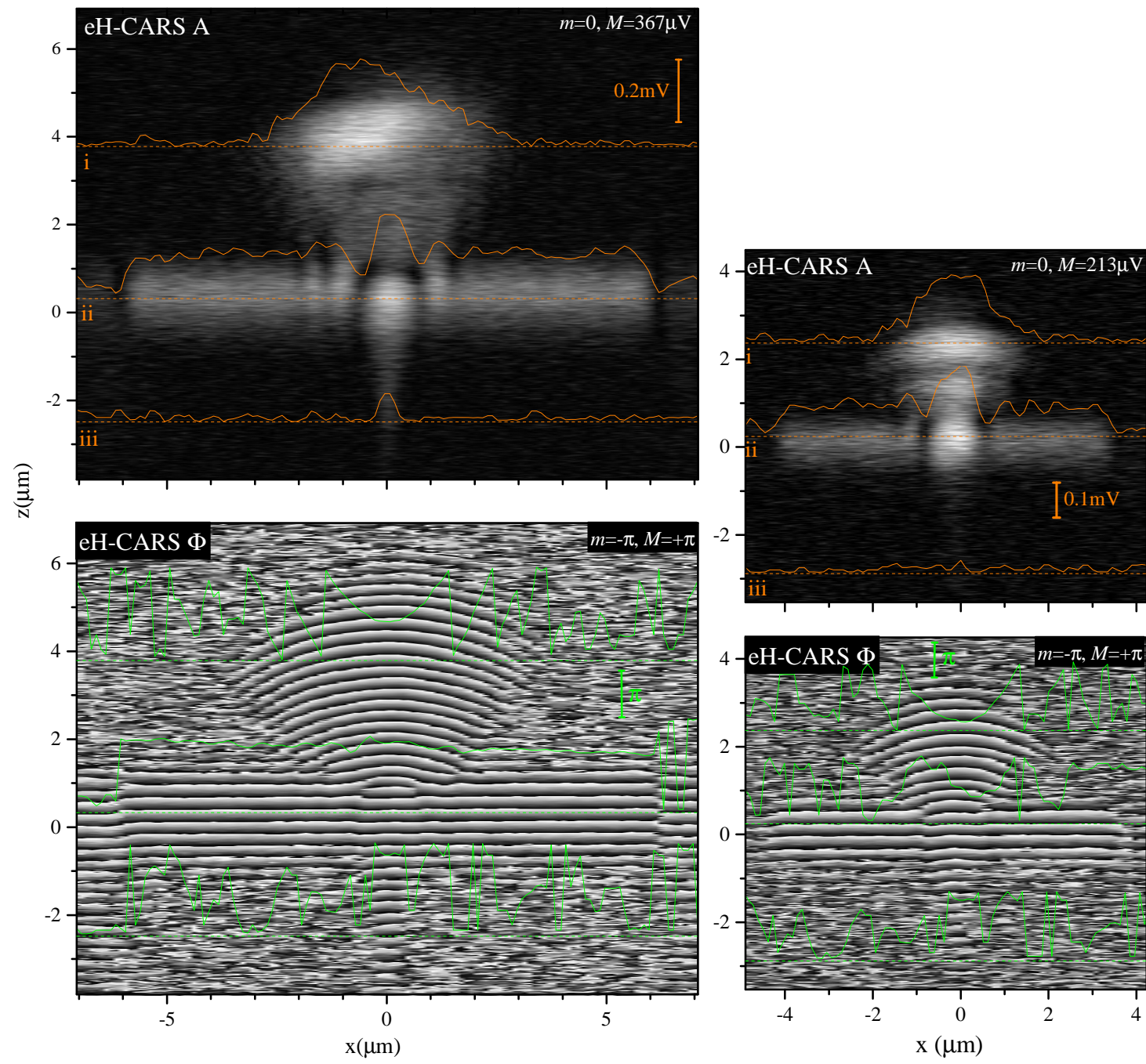

$m=0$
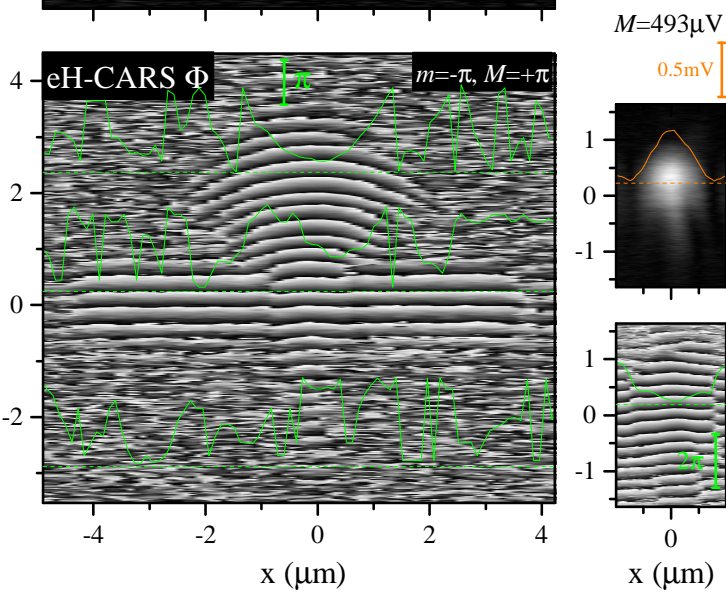

Figure 3. eH-CARS of oil droplets on a glass-water $(0.25 \%$ agar $)$ interface. eH-CARS amplitude $(\mathrm{A})$ and phase $(\Phi)$ in the $x z$ plane through the center in $y$ of oil droplets of about $12 \mu \mathrm{m}$ (left), $7 \mu \mathrm{m}$ (middle), and $1 \mu \mathrm{m}$ (right) in-plane diameter, which are indicated in Fig. 1 by the corresponding labels. Grayscale (see Fig. 6) from $m$ (black) to $M$ (white), with $m$ and $M$ as given. Data at selected values of $z$, indicated by dashed lines, and labelled i, ii, and iii, are given as curves, with the respective dashed line indicating zero, and a vertical scale as shown by the scale bar.

Moving the focus from bottom to top, we first find a signal as shown in Fig. 2a, where the focus is in the glass, creating a CARS signal. The CARS signal then propagates into the oil droplet, which has a similar refractive index as glass $n_{\mathrm{g}}=1.52$, resulting in negligible reflection or refraction at the glass-oil interface. The CARS is then reflected by the oil-water interface, having an amplitude reflection coefficient of $\left(n_{\mathrm{g}}-n_{\mathrm{w}}\right) /\left(n_{\mathrm{g}}+n_{\mathrm{w}}\right) \approx 0.07$ using the refractive index of water $n_{\mathrm{w}}=1.33$. Since the signal is detected as interference with the reference, measuring the product of the CARS field with the reference field, it represents a confocal detection. The reflection from the surface thus has to refocus the CARS signal into its point of creation, which requires the excitation focus to be at the center of the droplet curvature (using ray optics). Moving upwards, the next pathway, shown in Fig. 2b, is for a focus at the glass-oil interface, generating CARS propagating backwards by the step in $\chi^{(3)}$, between glass and oil. The next and final pathway, shown in Fig. 2c, is occuring for a focus at the oil-water interface. Here, there are two overlapping signal sources. Firstly, backward CARS is created by the step in $\chi^{(3)}$ between oil and water. Secondly, the forward CARS generated in oil is reflected at the oil-water interface, by the step in $\chi^{(1)}$.

Let's now move to the experimental data. The measured eH-CARS data in the $x z$ plane, with the axial 


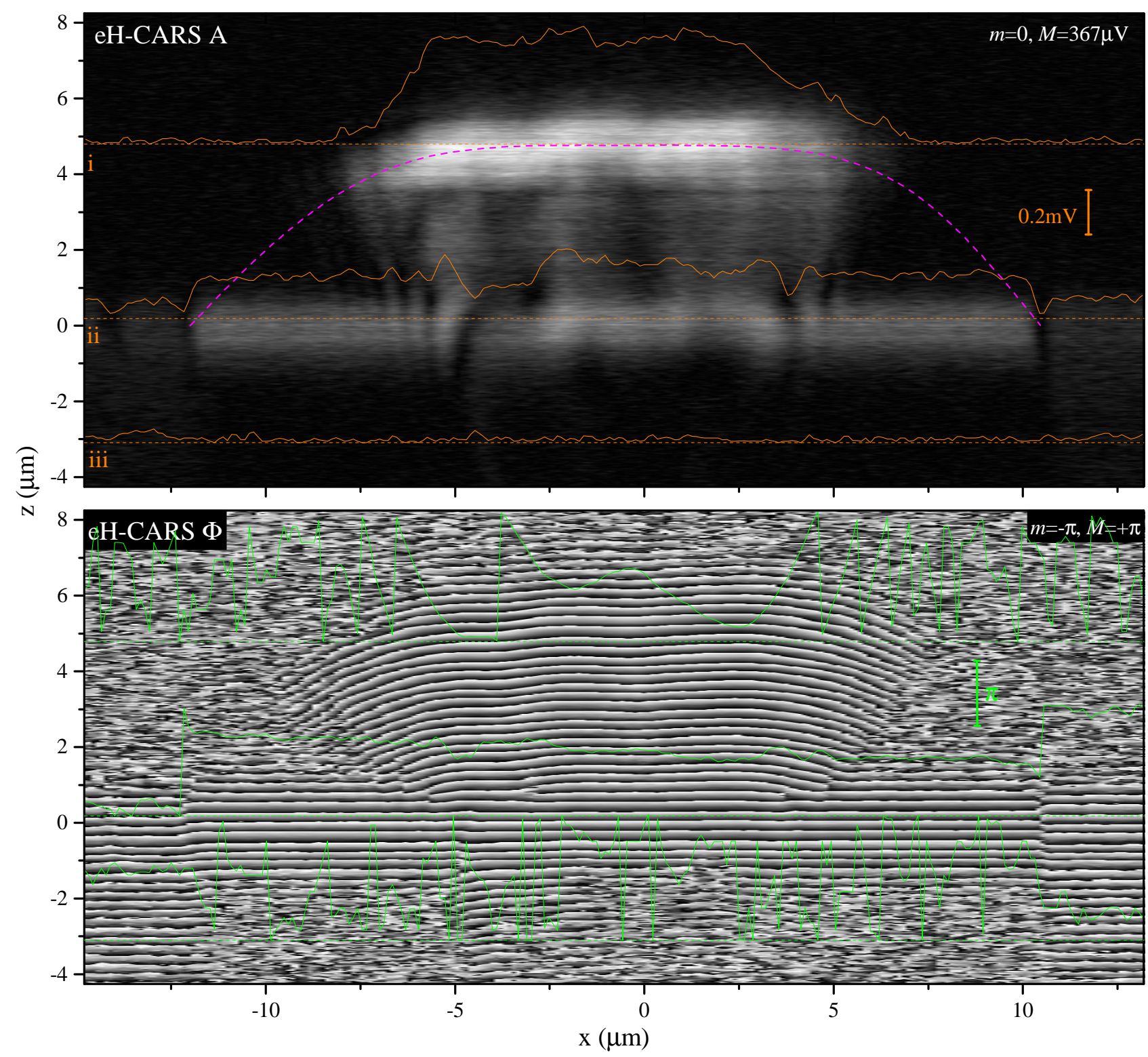

Figure 4. As Fig. 3, but for a large silicone oil droplet of about $20 \mu \mathrm{m}$ diameter, as indicated in Fig. 1 by the corresponding label. The magenta dashed line indicates the approximate shape of the oil-water interface. 
direction $z$, are shown in Fig. 3 for three lipid droplets of diameters at the interface of about $12 \mu \mathrm{m}, 7 \mu \mathrm{m}$, and $1 \mu \mathrm{m}$. The $y$ coordinate is chosen at the center of the droplet, so that the data are representing center crosssections. Looking at the largest droplet (Fig. 3 left), we see that the amplitude shows features in qualitative agreement with the discussed pathways in Fig. 2. Pathway (a), where the reflection of the CARS signal from the droplet top surface provides a signal at the droplet center around $x=0 \mu \mathrm{m}$, is visible as elongated stripe. The elongation along $z$ is attributed to the shape of the surface, which is not matching the CARS wavefront over the full angular range and thus creates aberrations in the back-reflection. The cut at $z=-2.5 \mu \mathrm{m}$ (cut iii) crosses the tail of the signal from this pathway, while at the maximum signal around $z=-0.5 \mu \mathrm{m}$, it interferes with pathway (b) at the glass surface at $z=0 \mu \mathrm{m}$ (cut ii), where the glass-oil interface (or glass-water interface for $|x| \gtrsim 6 \mu \mathrm{m}$ ) provides a signal. Off-center, e.g. at $x=4 \mu \mathrm{m}$, pathway (a) is negligible, so that pathway (b) is isolated. The non-linearity of the CARS generation and the confocal selection provides a $z$ sectioning with about $1 \mu \mathrm{m}$ full width at half maximum (FWHM). Pathway (c), the oil-water interface signal, is visible at $z=3.8 \mu \mathrm{m}$ (cut i). Notably, the curvature of the interface results in a loss of the interface signal away from the center of the droplet, at $|x| \gtrsim 4 \mu \mathrm{m}$, as the signal is reflected into off-axis directions not collected by the objective.

The phase shows the curvature of the oil-water interface, specifically for cut i. Furthermore, the phase of the signal from the glass-oil and glass-water interface for pathway (b), in cut ii. shows a change between the two interfaces, of about $\pi / 2$, which is related to the phase of the complex step in $\chi^{(3)}$. While oil is dominated by a resonant response of the $\mathrm{C}-\mathrm{H}$ vibrations, leading to a large imaginary part of $\chi^{(3)}$, water and glass are dominated by a non-resonant response, leading to a mostly real $\chi^{(3)}$. We thus expect a phase difference of the signal from the two interfaces of around $\pi / 2$, as observed. A detailed analysis of this phase, also as function of the IFD, is beyond the scope of this work, and will be reported elsewhere. Moving to the droplet of about $7 \mu \mathrm{m}$ size (Fig. 3 middle), the features discussed for the $12 \mu \mathrm{m}$ droplet are reproduced. Notably, the smaller size seems to provide a more symmetric surface shape, which can be attributed to the higher stiffness at larger curvature by the surface tension. For the smallest droplet of about $1 \mu \mathrm{m}$ size (Fig. 3 right), the three different pathways are overlapping within the spatial resolution of the eH-CARS imaging.

The effect of a non-spherical surface shape is specifically prominent for the large droplet of about $20 \mu \mathrm{m}$ diameter, shown in Fig. 4. For this large droplet the surface shows a modulated flat top, as can be seen in the phase of cut i, likely due to forces exerted by the agarose gel. This modulation results in a varying focussing of the signal of pathway (a), resulting in the vertical stripes in the amplitude. The phase-step of pathway (b) in cut ii is again observed, here slightly above $\pi / 2$.

Full three-dimensional eH-CARS data were taken on different regions of the sample, as stacks of planar $x y$ images. We show in Fig. 5 data from two regions, at the axial positions $z=0$ at the glass surface. The complex field is shown, encoded as amplitude and phase corresponding to value and hue of the image color. The left image shows the large droplet of Fig. 4. The phase change of pathway (b) between the glass-water and glass-oil interface is seen as the color change from green to blue, and the remaining interference of the signal from pathway (a) is seen in the center region, where the fringes show the varying height of the droplet. A region of smaller droplets is shown in the right image. The largest droplet of about $7 \mu \mathrm{m}$ diameter shows the same features as the large droplet on the left, but with a more symmetric interference pattern revealing the more spherical shape of the oil-water interface. Furthermore, a large number of smaller droplets with sizes below $4 \mu \mathrm{m}$ are observed. Interestingly, the interference between pathways (a) and (b) is interferometrically depending on the droplet height, resulting in a variety of phase and amplitude patterns. Only the smallest droplets show a rather uniform signal phase, which is attributed to a thickness much thinner than a wavelength. Detailed modelling of this response is presently under development and will be reported in a future work.

\subsection{Planar lipid bilayers}

We here show eH-CARS data from an important biophysical sample, namely lipid bilayers, extending what has been shown in Ref. 18. Synthetic lipid membrane bilayers have been investigated for several decades to study lipid-lipid and lipid-protein interactions ${ }^{22}$ and are model systems to aid our understanding of the heterogeneous organization of cellular membranes. Measuring the chemical composition and structure of lipid bilayers with high sensitivity and spatial resolution is thus important, yet technically challenging. 


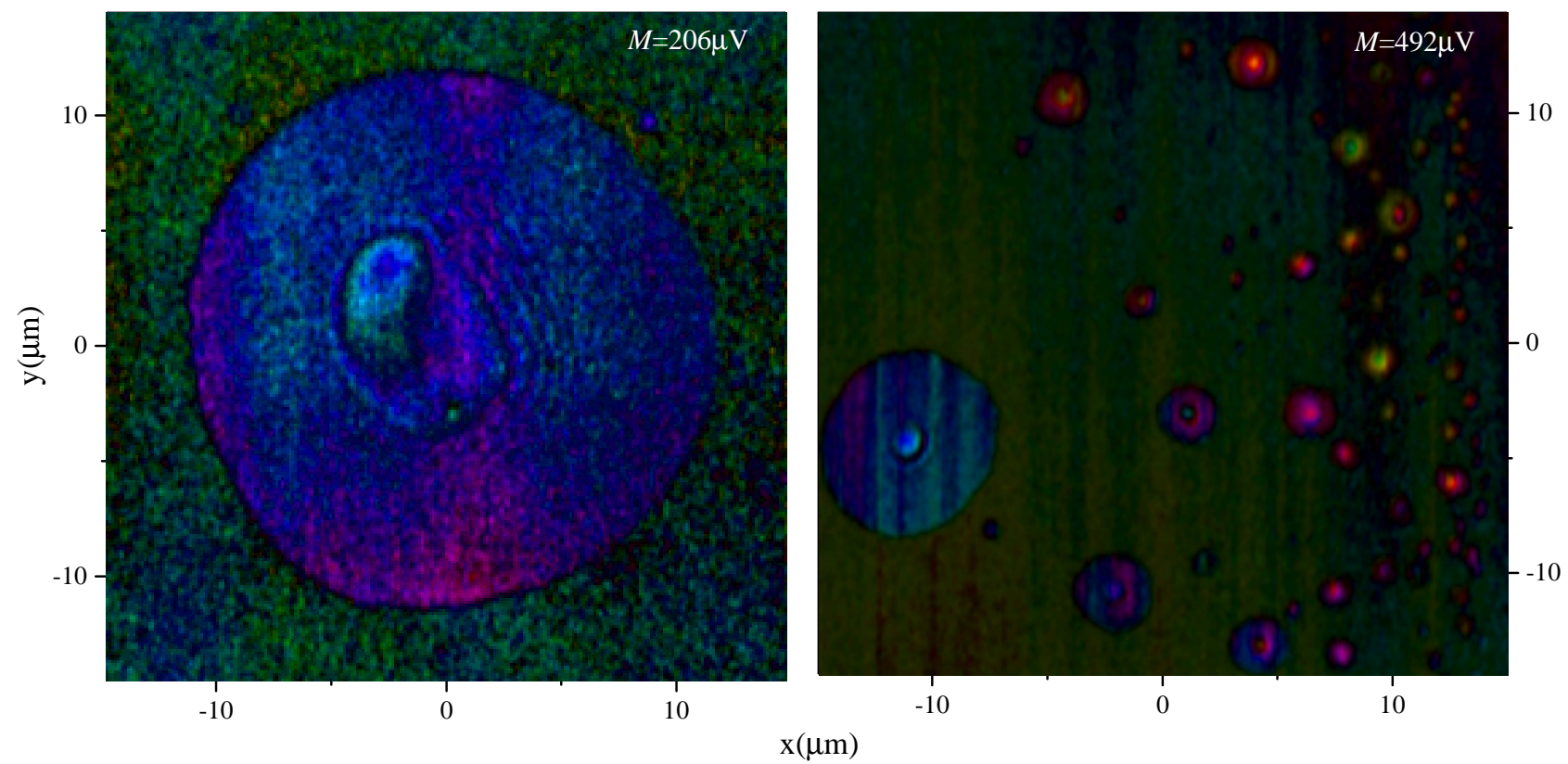

Figure 5. eH-CARS of oil droplets on a glass-water ( $0.25 \%$ agar $)$ interface. The eH-CARS complex field in the $x y$ plane is shown for an axial position at the glass surface $z=0 \mu \mathrm{m}$. The amplitude and phase are encoded in a value-hue color scale as given in Fig. 6. The maximum $M$ of the amplitude scale is given for each image. Two different regions are shown, left: containing the $20 \mu \mathrm{m}$ lipid droplet, right: region $\mathrm{C}$ indicated in Fig. 1, containing smaller lipid droplets. Note that some of the smaller droplets seems to have changed in the time between taking the two images.

Since the coherent signal enhancement in CRS compared to spontaneous Raman scattering relies on the constructive interference of the scattered light from many identical bonds in the focal volume, detection of a single 2D lipid bilayer, as compared to a 3D volume, pushes CRS microscopy to its sensitivity limits. ${ }^{2,23}$ Here we demonstrate eH-CARS on a single lipid bilayer. The sample consists of a single lipid type (1,2-dipentadecanoylsn-glycero-3-phosphocholine- $\mathrm{DC}_{15} \mathrm{PC}$ ) deposited onto a planar glass substrate in order to form regions with single and double bilayers. ${ }^{18}$ Supplementing the data in Ref. 18, we show here in Fig. 6 the eH-CARS on a region exibiting patches of zero (0BL), one (1BL), and two (2BL) bilayers. An IFD of $2840 \mathrm{~cm}^{-1}$ was used, near the peak of the $\mathrm{CH}_{2}$ symmetric stretch vibration of lipids. The data is shown in amplitude and phase separately (left column), and as complex field, with amplitude and phase given by value and hue of the image color, in the top right. A clear contrast is obtained distinguishing regions of different bilayer number. The amplitude changes from $0 \mathrm{BL}$ to $1 \mathrm{BL}$ by a factor of about 1.6, so that the phase of the signal is still dominated by the 0BL signal from the glass-water interface. The phase has been offset to result in a 0BL signal of close to zero phase.

In order to highlight the signal from the lipid bilayers, the data, offset by the 0BL signal, is shown in the middle of the right column. It is interesting to observe that the phase of the second bilayer is different from the phase of the first bilayer, as is clear from the color change. The phase difference can be attributed to the sensitivity of the epi-detected signal phase to the axial position of the signal source, similar to what we recently showed by measuring the heterodyne epi-detected resonant four-wave mixing of single gold nanoparticles.$^{24}$ The phase slope can be estimated as $k_{\text {signal }}+2 k_{\text {Pump }}-k_{\text {Stokes }}$ for plane wave excitation, resulting in $1.64^{\circ} / \mathrm{nm}$, using the glass refractive index of 1.518, and the wavelengths $665 \mathrm{~nm}, 820 \mathrm{~nm}, 1069 \mathrm{~nm}$ for signal, Pump, and Stokes beam, respectively. The phase slope measured in the experiment by moving the sample was found to be $(1.4 \pm 0.2)^{\circ} / \mathrm{nm}$, somewhat smaller than the above estimate as expected due to the Gouy phase shift in the focus. Using the measured phase slope, the observed phase difference corresponds to an axial position difference of $20 \mathrm{~nm}$ between first and second bilayer. Since the bilayer thickness is about $5 \mathrm{~nm}$, as confirmed by the qDIC measurements, this suggests the presence of an additional water layer thickness of about $15 \mathrm{~nm}$ between the two bilayers in the investigated sample region.

Furthermore, while the 1BL regions show a rather homogeneous phase, indicating that the first bilayer distance 

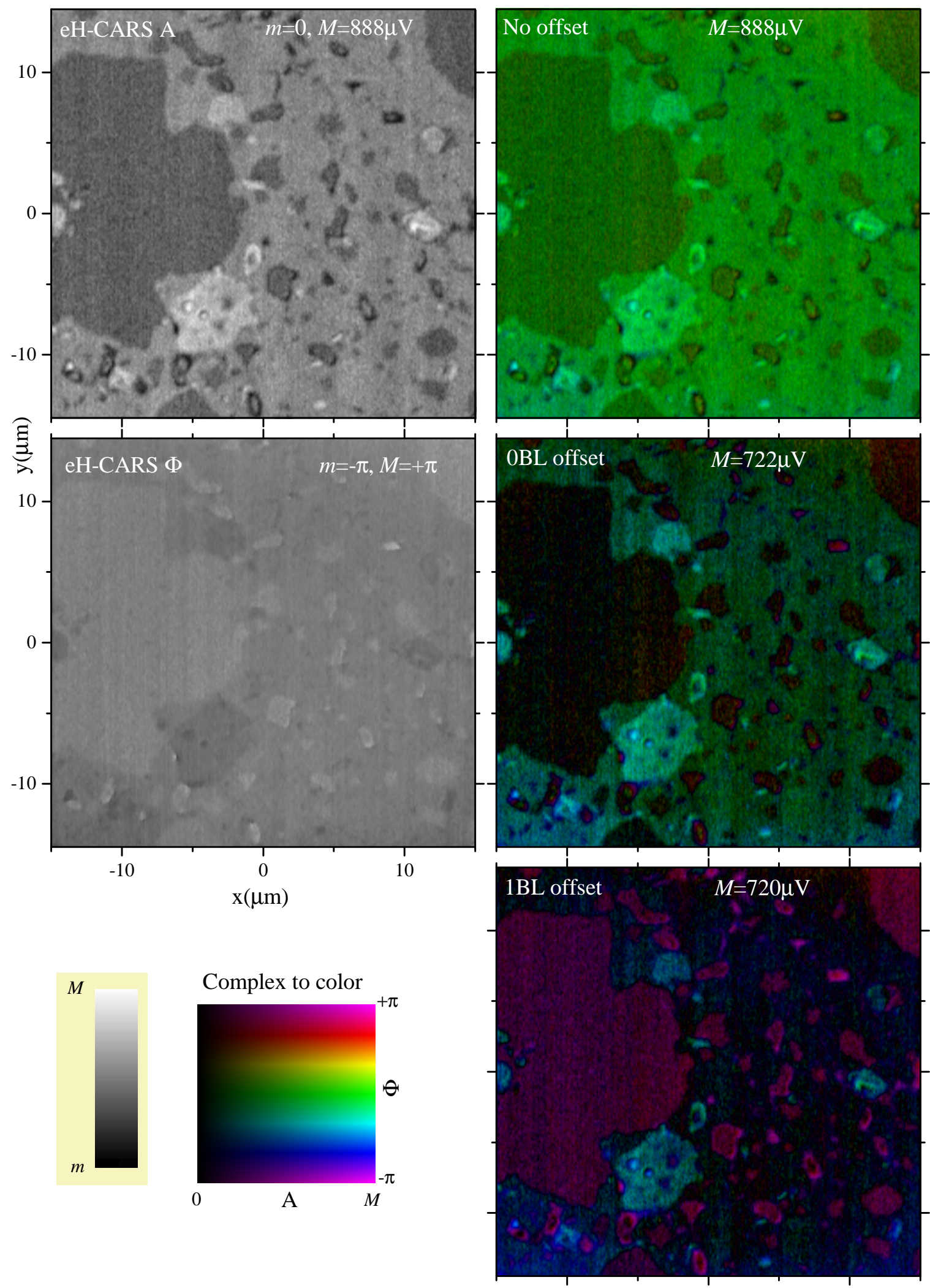

Figure 6. eH-CARS on a lipid bilayer sample measured at an IFD of $2840 \mathrm{~cm}^{-1}$. Same original data as used in Ref. 18 Fig.7. The amplitude $(\mathrm{A})$ and phase $(\Phi)$ in the $x y$ plane for an axial position at the interface is given on the left column. Regions without lipid (0BL), with one bilayer (1BL), and two bilayers are visible. In the right column the complex signal is encoded in a value-hue scale as given, as is (top), with a complex offset given by the 0BL signal (middle), and with a complex offset given by the 1BL signal (bottom). 
to the glass is well defined, and thus expected to be below a few nanometers, 2BL regions show significant phase variations. In order to highlight the phase of the $2 \mathrm{BL}$ signal, we offset the measured signal by the $1 \mathrm{BL}$ signal, as shown in the bottom of the right column. Clearly, while the 0BL regions have a well defined phase, given by the purple color, the 2BL regions have a varying phase, reaching from cyan to blue. Since the bilayer thickness is well defined, these variations are attributed to height variations. The observed color change corresponds to about $70^{\circ}$ phase change, corresponding to $50 \mathrm{~nm}$ axial change of the second bilayer. These results exemplify the added capabilities of phase-sensitive eH-CARS compared to an intensity based detection, not only to distinguish and properly subtract non-resonant backgrounds, but also to extract additional information related to the topography of the sample. Note that, in general, without additional information, phase changes due to axial position changes cannot be separated from those due to susceptibility changes. However, this can be resolved by measuring at different IFDs.

\section{CONCLUSION}

We have built and demonstrated epi-heterodyne CARS with balanced diode detection, which offers backgroundfree chemically-specific image contrast, shot-noise limited detection, and phase sensitivity. Investigating lipid droplets on a glass surface in water, we have identified three pathways of eH-CARS signal generation, which were exemplified using droplets of different size. The phase sensitivity allows to determine the shape of the droplet surface with high resolution, in the nanometer range. The relevance of the phase-sensitive measurement is furthermore shown on a planar single lipid bilayer, pushing CRS microscopy to its detection limits. Our results suggest that with this modality we not only can resolve a single lipid bilayer, distinct from a double bilayer, and measure the phase of its susceptibility, but also extract additional phase information to determine the topography of the bilayer with nanometer resolution. Information on the data underpinning the results presented here, including how to access them, can be found in the Cardiff University data catalogue at http://doi.org/10.17035/d.2019.0067933588.

\section{ACKNOWLEDGMENTS}

This work was supported by the UK Engineering and Physical Science Research Council (Grant n. EP/I005072/1, EP/I016260/1, EP/M028313/1, EP/M507842/1). PB acknowledges the Royal Society for her Wolfson research merit award (grant WM140077).

\section{REFERENCES}

[1] Evans, C. L. and Xie, X. S., "Coherent anti-stokes raman scattering microscopy: Chemical imaging for biology and medicine," Annu. Rev. Anal. Chem. 1, 883-909 (2008).

[2] Zumbusch, A., Langbein, W., and Borri, P., "Nonlinear vibrational microscopy applied to lipid biology," Progress in Lipid Research 52, 615-632 (2013).

[3] Zhang, C. and Cheng, J.-X., "Perspective: Coherent raman scattering microscopy, the future is bright," APL Photonics 3, 090901 (sep 2018).

[4] Pezacki, J. P., Blake, J. A., Danielson, D. C., Kennedy, D. C., Lyn, R. K., and Singaravelu, R., "Chemical contrast for imaging living systems: molecular vibrations drive cars microscopy," Nat. Chem. Biol. 7, 137145 (2011).

[5] Paar, M., Jüngst, C., Steiner, N. A., Magnes, C., Sinner, F., Kolb, D., Lass, A., Zimmermann, R., Zumbusch, A., Kohlwein, S. D., and Wolinski, H., "Remodeling of lipid droplets during lipolysis and growth in adipocytes," J Biol Chem 287(14), 11164-73 (2012).

[6] Bradley, J., Pope, I., Masia, F., Sanusi, R., Langbein, W., Swann, K., and Borri, P., "Quantitative imaging of lipids in live mouse oocytes and early embryos using cars microscopy," Development 143(12), 2238-2247 (2016).

[7] Di Napoli, C., Pope, I., Masia, F., Langbein, W., Watson, P., and Borri, P., "Quantitative spatiotemporal chemical profiling of individual lipid droplets by hyperspectral cars microscopy in living human adiposederived stem cells," Anal. Chem. 88(7), 3677-3685 (2016). 
[8] Cheng, J.-X. and Xie, X., "Coherent anti-stokes raman scattering microscopy: Instrumentation, theory, and applications," J. Phys. Chem. B 108(3), 827-840 (2004).

[9] Rinia, H. A., Burger, K. N. J., Bonn, M., and Müller, M., "Quantitative label-free imaging of lipid composition and packing of individual cellular lipid droplets using multiplex cars microscopy," Biophys. J. 95, 4908-4914 (2008).

[10] Camp, C. H., Lee, Y. J., Heddleston, J. M., Hartshorn, C. M., Walker, A. R. H., Rich, J. N., Lathia, J. D., and Cicerone, M. T., "High-speed coherent raman fingerprint imaging of biological tissues," Nat. Phot. 8, 627-634 (2014).

[11] Masia, F., Glen, A., Stephens, P., Borri, P., and Langbein, W., "Quantitative chemical imaging and unsupervised analysis using hyperspectral coherent anti-stokes raman scattering microscopy," Anal. Chem. 85(22), 10820-10828 (2013).

[12] Napoli, C. D., Pope, I., Masia, F., Watson, P., Langbein, W., and Borri, P., "Hyperspectral and differential cars microscopy for quantitative chemical imaging in human adipocytes," Biomed. Opt. Express 5, 1378-1390 (May 2014).

[13] Masia, F., Glen, A., Stephens, P., Langbein, W., and Borri, P., "Label-free quantitative chemical imaging and classification analysis of adipogenesis using mouse embryonic stem cells," J. Biophotonics 11, e201700219 (2018).

[14] Masia, F., Pope, I., Watson, P., Langbein, W., and Borri, P., "Bessel-beam hyperspectral cars microscopy with sparse sampling: Enabling high-content high-throughput label-free quantitative chemical imaging," Anal. Chem. 90, 3775-3785 (2018).

[15] Evans, C. L., Potma, E. O., and Xie, X. S., "Coherent anti-stokes raman scattering spectral interferometry: determination of the real and imaginary components of nonlinear susceptibility $\chi(3)$ for vibrational microscopy," Opt. Lett. 29, 2923-2925 (2004).

[16] Potma, E. O., Evans, C. L., and Xie, X. S., "Heterodyne coherent anti-stokes raman scattering (cars) imaging," Opt. Lett. 31, 241-243 (Jan 2006).

[17] Jurna, M., Korterik, J. P., Otto, C., Herek, J. L., and Offerhaus, H. L., "Vibrational phase contrast microscopy by use of coherent anti-stokes raman scattering," Phys. Rev. Lett. 103, 043905 (Jul 2009).

[18] Langbein, W., Regan, D., Pope, I., and Borri, P., "Heterodyne dual-polarization epi-detected cars microscopy for chemical and topographic imaging of interfaces," APL Photonics 3, 092402 (sep 2018).

[19] Rocha-Mendoza, I., Langbein, W., and Borri, P., "Coherent anti-stokes raman microspectroscopy using spectral focusing with glass dispersion," Appl. Phys. Lett. 93, 201103 (2008).

[20] Langbein, W., Rocha-Mendoza, I., and Borri, P., "Single source coherent anti-stokes raman microspectroscopy using spectral focusing," Appl. Phys. Lett. 95(8), 081109 (2009).

[21] Langbein, W., Rocha-Mendoza, I., and Borri, P., "Coherent anti-stokes raman micro-spectroscopy using spectral focusing: Theory and experiment," J. Raman Spectrosc. 40, 800-808 (2009).

[22] Bagatolli, L. A., Ipsen, J. H., Simonsen, A. C., and Mouritsen, O. G., "An outlook on organization of lipids in membranes: Searching for a realistic connection with the organization of biological membranes," Prog. Lipid Res. 49, 378-389 (2010).

[23] Li, L. and Cheng, J.-X., "Label-free coherent anti-stokes raman scattering imaging of coexisting lipid domains in single bilayers," J. Phys. Chem. B 112, 1576-1579 (2008).

[24] Zoriniants, G., Masia, F., Giannakopoulou, N., Langbein, W., and Borri, P., "Background-free 3d nanometric localization and sub-nm asymmetry detection of single plasmonic nanoparticles by four-wave mixing interferometry with optical vortices," Phys. Rev. X 7, 041022 (2017). 